International Journal of Pure and Applied Mathematics

Volume 87 No. 2 2013, 333-347

ISSN: 1311-8080 (printed version); ISSN: 1314-3395 (on-line version)

url: http://www.ijpam.eu

doi: http://dx.doi.org/10.12732/ijpam.v87i2.14

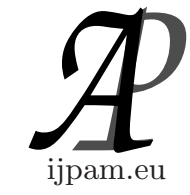

\title{
SOME COMMON FIXED POINT THEOREMS USING IMPLICIT RELATIONS IN FUZZY METRIC SPACES
}

Shin Min Kang ${ }^{1}$, Vishal Gupta ${ }^{2}$, Balbir Singh ${ }^{3}$, Sanjay Kumar ${ }^{4}$

${ }^{1}$ Department of Mathematics and RINS

Gyeongsang National University

Jinju, 660-701, KOREA

${ }^{2}$ Department of Mathematics

Maharishi Markandeshwar University

Mullana 133203, INDIA

${ }^{3}$ Department of Mathematics

Bhagwan Mahaveer Institute of Engineering and Technology

Sonipat, 131001, INDIA

${ }^{4}$ Department of Mathematics

Deenbandhu Chhotu Ram University of Science and Technology

Murthal, 131039, INDIA

Abstract: In this paper, we prove common fixed point theorems for pairs of weakly compatible mappings

AMS Subject Classification: 47H10, 54H 25

Key Words: fuzzy metric spaces, weakly compatible mappings, $t$-norm of Hadžić-type, E.A. property and CLR property

\section{Introduction}

It proved a turning point in the development of fuzzy mathematics, when the notion of fuzzy set was introduced by Zadeh [20]. Fuzzy set theory has many applications in applied science such as neural network theory, stability theory, mathematical programming, modelling theory, engineering sciences, medical

Received: June 3, 2013

(c) 2013 Academic Publications, Ltd.

$\S_{\text {Correspondence author }}$ url: www.acadpubl.eu 
sciences (medical genetics, nervous system), image processing, control theory, communication, etc. There are many view points of the notion of the fuzzy metric spaces in fuzzy topology (see Deng [2], Erceg [3], George and Veermani [4], Kaleva and Seikkala [12], Kramosil and Michalek [13]).

In this paper, we are considering the fuzzy metric space in the sence of Kramosil and Michalek.

Definition 1.1. A binary operation $\Delta$ on $[0,1]$ is a $t$-norm if it satisfies the following conditions:

(i) $\Delta$ is associative and commutative,

(ii) $\Delta(a, 1)=a$ for every $a \in[0,1]$,

(iii) $\Delta(a, b) \leq \Delta(c, d)$, whenever $a \leq c$ and $b \leq d$.

Basics examples of $t$-norm are the Lukasiewicz $t$-norm $\Delta_{L}, \Delta_{L}(a, b)=$ $\max \{a+b-1,0\}$, the product $t$-norm $\Delta_{P}, \Delta_{P}(a, b)=a b$ and the minimum $t$-norm $\Delta_{M}, \Delta_{M}(a, b)=\min \{a, b\}$.

Definition 1.2. ([6]) Let $\Delta$ be a $t$-norm and $\Delta_{n}:[0,1] \rightarrow[0,1](n \in \mathbb{N})$ be defined by

$$
\Delta_{1}(x)=\Delta(x, x), \quad \Delta_{n+1}(x)=\Delta\left(\Delta_{n}(x), x\right) \quad(n \in \mathbb{N}, x \in[0,1]) .
$$

Then we say that the $t$-norm $\Delta$ is of Hadžić-type if the family $\left\{\Delta_{n}(x) ; n \in \mathbb{N}\right\}$ is equicontinuous at $x=1$. The family $\left\{\Delta_{n}(x) ; n \in \mathbb{N}\right\}$ is said to be equicontinuous at $x=1$ if for every $\lambda \in(0,1)$, there exists $\delta(\lambda) \in(0,1)$ such that

$$
x>1-\delta(\lambda) \text { implies } \Delta_{n}(x)>1-\lambda \quad(n \in \mathbb{N}) .
$$

A trivial example of $t$-norm of Hadžić-type is $\Delta=\Delta_{M}$.

Remark 1.3. ([7]) (1) If there exists a strictly increasing sequence $\left\{b_{n}\right\}_{n \in \mathbb{N}}$ in $[0,1]$ such that $\lim _{n \rightarrow \infty} b_{n}=1$ and $\Delta\left(b_{n}, b_{n}\right)=b_{n}$ for all $n \in \mathbb{N}$, then $\Delta$ is of Hadžić-type.

(2) If $\Delta$ is continuous and of Hadžić-type, then there exists a sequence $\left\{b_{n}\right\}_{n \in \mathbb{N}}$ as in (1).

Definition 1.4. ([7]) If $\Delta$ is a $t$-norm and $\left(x_{1}, x_{2}, x_{3}, \ldots, x_{n}\right) \in[0,1]^{n}(n \in$ $\mathbb{N})$, then $\Delta_{i=1}^{n} x_{i}$ is defined recurrely by 1 if $n=1$ and $\Delta_{i=1}^{n} x_{i}=\Delta\left(\Delta_{i=1}^{n-1} x_{i}, x_{n}\right)$ for all $n \geq 2$. If $\left\{x_{n}\right\}_{n \in \mathbb{N}}$ is a sequence in $[0,1]$, then $\Delta_{i=1}^{\infty} x_{i}$ is defined as $\lim _{n \rightarrow \infty} \Delta_{i=1}^{n} x_{i}$ (this limit always exists) and $\Delta_{i=1}^{\infty} x_{i}$ as $\Delta_{i=1}^{\infty} x_{n+i}$.

Definition 1.5. The 3 -triple $(X, M, \Delta)$ is called a fuzzy metric space in the sence of Kramosil and Michalek if $X$ is an arbitrary set, $\Delta$ is a continuous $t$-norm and $M$ is a fuzzy set on $X^{2} \times[0, \infty)$ satisfying the following conditions: for all $x, y, z \in X$ and $s, t>0$ 
$(\mathrm{FM}-1) M(x, y, 0)=0$,

(FM-2) $M(x, y, t)=1$ for all $t>0$ if and only if $x=y$,

(FM-3) $M(x, y, t)=M(y, x, t)$,

(FM-4) $M(x, z, t+s) \geq \Delta(M(x, y, t), M(y, z, s))$,

$($ FM-5) $M(x, y, \cdot):[0, \infty) \rightarrow[0,1]$ is left continuous.

Note that $M(x, y, t)$ can be thought of as the degree of nearness between $x$ and $y$ with respect to $t$. We identify $x=y$ with $M(x, y, t)=1$ for all $t>0$ and $M(x, y, t)=0$ with $t=0$. Since $\Delta$ is a continuous $t$-norm, it follows from (FM-4) that the limit of the sequence in fuzzy metric space is uniquely determined.

Definition 1.6. A sequence $\left\{x_{n}\right\}$ in a fuzzy metric space $(X, M, \Delta)$ is said to be

(1) convergent to the $\operatorname{limit} x$ if $\lim _{n \rightarrow \infty} M\left(x_{n}, x, t\right)=1$ for all $t>0$.

(2) Cauchy sequence in $X$ if for every $\lambda \in(0,1)$ and $t>0$, there exists a positive integer $N$ such that $M\left(x_{n}, x_{m}, t\right)>1-\lambda$ whenever $m, n \geq N$.

(3) complete if every Cauchy sequence in $X$ is convergent in $X$.

Fixed point theory in fuzzy metric spaces has been developing since the paper of Grabiec [5]. Subramanyam [18] gave a generalization of Jungck's theorem ([10]) for commuting mapping in the setting of fuzzy metric spaces.

In 1994, Pant [16] introduced the concept of $R$-weakly commuting mappings in metric spaces. Later on, Vasuki [19] initiated the concept of non-compatible of mappings in fuzzy metric spaces and introduced the notion of $R$-weakly commuting mappings in fuzzy metric spaces and proved some common fixed point theorems for these mappings.

Definition 1.7. Let $f$ and $g$ be self-mappings on a fuzzy metric space $(X, M, \Delta)$. Then a pair $(f, g)$ is said to be

(1) weakly commuting if

$$
M(f g x, g f x, t) \geq M(f x, g x, t)
$$

for all $x \in X$ and $t>0$,

(2) $R$-weakly commuting if there exists $R>0$ such that

$$
M(f g x, g f x, t) \geq M(f x, g x, t / R)
$$

for all $x \in X$ and $t>0$.

In 1994, Mishra et al. [15] generalized the notion of weakly commuting to compatible mappings in fuzzy metric spaces. 
Definition 1.8. Let $f$ and $g$ be self-mappings on a fuzzy metric space $(X, M, \Delta)$. Then a pair $(f, g)$ is said to be compatible if

$$
\lim _{n \rightarrow \infty} M\left(f g x_{n}, g f x_{n}, t\right)=1
$$

whenever $\left\{x_{n}\right\}$ is a sequence in $X$ such that $\lim _{n \rightarrow \infty} f x_{n}=\lim _{n \rightarrow \infty} g x_{n}=u$ for some $u \in X$ and for all $t>0$.

Definition 1.9. Let $f$ and $g$ be self-mappings on a fuzzy metric space $(X, M, \Delta)$. Then a pair $(f, g)$ is said to be non-compatible if

$$
\lim _{n \rightarrow \infty} M\left(f g x_{n}, g f x_{n}, t\right) \neq 1
$$

whenever $\left\{x_{n}\right\}$ is a sequence in $X$ such that $\lim _{n \rightarrow \infty} f x_{n}=\lim _{n \rightarrow \infty} g x_{n}=u$ for some $u \in X$ and for all $t>0$.

In 1996, Jungck [11] introduced the notion of weakly compatible.

Definition 1.10. Two mappings $f$ and $g$ are said to be weakly compatible if they commute at their coincidence points.

In 2002, Aamri and Moutawakil [1] generalized the notion of non-compatible mappings to E.A. property. It was pointed out in ([1]), that E.A. property buys containment of ranges without any continuity requirements besides minimizes the commutativity conditions of the mappings at their coincidence points. Moreover, E.A. property allows replacing the completeness requirement of the space with a more natural condition of closeness of the range. Recently, some common fixed point theorems in probabilistic metric spaces/fuzzy metric spaces using E.A. property with weak compatibility have been recently obtained in ([8], [9], [14]).

Definition 1.11. ([1]) Let $f$ and $g$ be self-mappings on a metric space $(X, d)$. Then a pair $(f, g)$ is said to satisfy E.A. property if there exists a sequence $\left\{x_{n}\right\}$ in $X$ such that $\lim _{n \rightarrow \infty} f x_{n}=\lim _{n \rightarrow \infty} g x_{n}=u$ for some $u \in X$.

Now, in a similar mode, we can state E.A. property in fuzzy metric space.

Definition 1.12. Let $f$ and $g$ be self-mappings on a fuzzy metric space $(X, M, \Delta)$. Then a pair $(f, g)$ is said to satisfy E.A. property if there exists a sequence $\left\{x_{n}\right\}$ in $X$ such that $\lim _{n \rightarrow \infty} M\left(f x_{n}, g x_{n}, t\right)=1$ for some $t \in X$.

Example 1.13. Let $X=[0, \infty)$ be the usual metric space. Define $f, g$ : $X \rightarrow X$ by $f x=x / 4$ and $g x=3 x / 4$ for all $x \in X$. Consider the sequence $\left\{x_{n}\right\}=1 / n$. Since $\lim _{n \rightarrow \infty} f x_{n}=\lim _{n \rightarrow \infty} g x_{n}=0$, therefore $f$ and $g$ satisfy the E.A. property. 
Although E.A property is generalization of the concept of non-compatible mappings yet it requires either completeness of the whole space or any of the range space or continuity of mappings. Recently the new notion of CLR property (common limit in the range property) was given by Sintunavarat and Kumam [17] that does not impose such conditions. The importance of CLR property ensures that one does not require the closeness of range subspaces.

Definition 1.14. ([17]) Let $f$ and $g$ be self-mappings on a fuzzy metric space $(X, M, \Delta)$. Then a pair $(f, g)$ is said to satisfy $C L R g$ property (common limit in the range of $g$ property) if $\lim _{n \rightarrow \infty} f x_{n}=\lim _{n \rightarrow \infty} g x_{n}=g x$ for some $x \in X$.

Example 1.15. Let $X=[0, \infty)$ be the usual metric space. Define $f, g$ : $X \rightarrow X$ by $f x=x+1$ and $g x=2 x$ for all $x \in X$. Consider the sequence $\left\{x_{n}\right\}=1+\frac{1}{n}$. Since $\lim _{n \rightarrow \infty} f x_{n}=\lim _{n \rightarrow \infty} g x_{n}=2=g 1$, therefore $f$ and $g$ satisfy the CLRg property.

Now we state a lemma which is useful in our work.

Lemma 1.16. ([15]) Let $(X, M, \Delta)$ be a fuzzy metric space. If there exists $q \in(0,1)$ such that $M(x, y, q t) \geq M(x, y, t)$ for all $x, y \in X$ and $t>0$, then $x=y$.

\section{Implicit Relations}

Let $\mathcal{F}$ be set of all continuous functions $F\left(t_{1}, t_{2}, t_{3}, t_{4}, t_{5}, t_{6}\right): \mathbb{R}^{6} \rightarrow \mathbb{R}$ is a non-increasing in 6 -th coordinate variable satisfying the following conditions:

(i) $F(u, 1, v, 1, v, \Delta(u, v)) \geq 1$ or $F(u, v, v, u, 1, \Delta(u, v)) \geq 1$ implies that $u \geq v$,

(ii) $F(u, 1,1, u, 1, u) \geq 1$ implies that $u \geq 1$,

(iii) $F(u, v, 1,1, v, u) \geq 1$ implies that $u \geq v$.

Example 1.17. Define $F\left(t_{1}, t_{2}, t_{3}, t_{4}, t_{5}, t_{6}\right)=15 t_{1}-13 t_{2}+5 t_{3}-7 t_{4}+t_{5}-t_{6}$. Then $F \in \mathcal{F}$.

\section{Main Theorems}

Now, we prove a fixed point thoeorem for weakly compatible mappings. 
Theorem 2.1. Let $(X, M, \Delta)$ be a complete fuzzy metric space with continuous t-norm of Hadžić-type. Let $A, B, S$ and $T$ be self-mappings on $X$ satisfying the following conditions:

(C1) $A(X) \subset T(X), B(X) \subset S(X)$,

$(C 2)$ the pairs $(A, S)$ and $(B, T)$ are weakly compatible,

(C3) there exists $q \in(0,1)$ such that for all $x, y \in X, t>0$ and $F \in \mathcal{F}$,

$$
\begin{aligned}
& F(M(A x, B y, q t), M(S x, T y, t), M(A x, S x, t), \\
& \quad M(B y, T y, q t), M(A x, T y, t), M(B y, S x,(q+1) t)) \geq 1,
\end{aligned}
$$

(C4) one of the subsets $A(X), B(X), S(X)$ and $T(X)$ is closed of $X$.

Assume that there exist $x_{0}, x_{1} \in X$ such that for $y_{1}=A x_{0}=T x_{1}, y_{2}=$ $B x_{1}=S x_{2}$ and $\mu \in(q, 1)$

$$
\lim _{n \rightarrow \infty} \Delta_{i=n}^{\infty} M\left(y_{1}, y_{2}, 1 / \mu^{i}\right)=1 .
$$

Then $A, B, S$ and $T$ have a unique common fixed point in $X$.

Proof. Since $B(X) \subset S(X)$, there exist $x_{1}, x_{2} \in X$ such that $B x_{1}=S x_{2}$. Inductively, we can construct two sequences $\left\{x_{n}\right\}$ and $\left\{y_{n}\right\}$ of $X$ such that

$$
y_{2 n-1}=T x_{2 n-1}=A x_{2 n-2}, \quad y_{2 n}=S x_{2 n}=B x_{2 n-1}
$$

for $n=1,2, \ldots$ Putting $x=x_{2 n}$ and $y=x_{2 n+1}$ in $(C 3)$, we have that for all $t>0$

$$
\begin{aligned}
1 \leq F & \left(M\left(A x_{2 n}, B x_{2 n+1}, q t\right), M\left(S x_{2 n}, T x_{2 n+1}, t\right), M\left(A x_{2 n}, S x_{2 n}, t\right),\right. \\
& M\left(B x_{2 n+1}, T x_{2 n+1}, q t\right), M\left(A x_{2 n}, T x_{2 n+1}, t\right), \\
& \left.M\left(B x_{2 n+1}, S x_{2 n},(q+1) t\right)\right) \\
= & F\left(M\left(y_{2 n+1}, y_{2 n+2}, q t\right), M\left(y_{2 n}, y_{2 n+1}, t\right), M\left(y_{2 n+1}, y_{2 n}, t\right),\right. \\
& M\left(y_{2 n+2}, y_{2 n+1}, q t\right), M\left(y_{2 n+1}, y_{2 n+1}, t\right), \\
& \left.M\left(y_{2 n+2}, y_{2 n},(q+1) t\right)\right) \\
\leq & F\left(M\left(y_{2 n+1}, y_{2 n+2}, q t\right), M\left(y_{2 n}, y_{2 n+1}, t\right), M\left(y_{2 n+1}, y_{2 n}, t\right),\right. \\
& M\left(y_{2 n+2}, y_{2 n+1}, q t\right), M\left(y_{2 n+1}, y_{2 n+1}, t\right), \\
& \left.\Delta\left(M\left(y_{2 n+2}, y_{2 n+1}, q t\right), M\left(y_{2 n+1}, y_{2 n}, t\right)\right)\right)
\end{aligned}
$$

since the function $F$ is non-increasing in the 6-th coordinate variable. Using properties of implicit relations $\mathcal{F}$, we get

$$
M\left(y_{2 n+1}, y_{2 n+2}, q t\right) \geq M\left(y_{2 n}, y_{2 n+1}, t\right) .
$$


Again, putting $x=x_{2 n+1}$ and $y=x_{2 n+2}$ in (C3), we have that for all $t>0$

$$
\begin{aligned}
1 \leq & F\left(M\left(A x_{2 n+1}, B x_{2 n+2}, q t\right), M\left(S x_{2 n+1}, T x_{2 n+2}, t\right),\right. \\
& M\left(A x_{2 n+1}, S x_{2 n+1}, t\right), M\left(B x_{2 n+2}, T x_{2 n+2}, q t\right), \\
& \left.M\left(A x_{2 n+1}, T x_{2 n+2}, t\right), M\left(B x_{2 n+2}, S x_{2 n+1},(q+1) t\right)\right) \\
= & F\left(M\left(y_{2 n+2}, y_{2 n+3}, q t\right), M\left(y_{2 n+1}, y_{2 n+2}, t\right), M\left(y_{2 n+2}, y_{2 n+1}, t\right),\right. \\
& M\left(y_{2 n+3}, y_{2 n+2}, q t\right), M\left(y_{2 n+2}, y_{2 n+2}, t\right), \\
& \left.M\left(y_{2 n+3}, y_{2 n+1},(q+1) t\right)\right) \\
\leq & F\left(M\left(y_{2 n+2}, y_{2 n+3}, q t\right), M\left(y_{2 n+1}, y_{2 n+2}, t\right), M\left(y_{2 n+2}, y_{2 n+1}, t\right),\right. \\
& M\left(y_{2 n+3}, y_{2 n+2}, q t\right), M\left(y_{2 n+2}, y_{2 n+2}, t\right), \\
& \left.\Delta\left(M\left(y_{2 n+3}, y_{2 n+2}, q t\right), M\left(y_{2 n+2}, y_{2 n+1}, t\right)\right)\right) .
\end{aligned}
$$

Hence we get

$$
M\left(y_{2 n+2}, y_{2 n+3}, q t\right) \geq M\left(y_{2 n+1}, y_{2 n+2}, t\right) .
$$

Thus, for any $n \in \mathbb{N}$, we have

$$
M\left(y_{n+1}, y_{n}, q t\right) \geq M\left(y_{n}, y_{n-1}, t\right)
$$

i.e.,

$$
\begin{aligned}
M\left(y_{n+1}, y_{n}, t\right) \geq & M\left(y_{n}, y_{n-1}, t / q\right) \\
\geq & M\left(y_{n-1}, y_{n-2}, t / q^{2}\right) \\
& \cdots \\
\geq & M\left(y_{1}, y_{2}, t / q^{n-1}\right) .
\end{aligned}
$$

Thus for all $t>0$ and $n=1,2,3, \ldots$

$$
M\left(y_{n}, y_{n+1}, q t\right) \geq M\left(y_{1}, y_{2}, t / q^{n-1}\right) .
$$

Now, we show that $\left\{y_{n}\right\}$ is a Cauchy sequence in $X$.

Let $\sigma=\frac{q}{\mu}$. Since $0<\sigma<1$, the series $\sum_{i=1}^{\infty} \sigma^{i}$ is convergent and there exists $m_{0} \in \mathbb{N}$ such that $\sum_{i=m_{0}}^{\infty} \sigma^{i}<1$. Hence for every $m>m_{0}+1$ and $s \in \mathbb{N}$

$$
t>t \sum_{i=m_{0}}^{\infty} \sigma^{i}>t \sum_{i=m-1}^{m+s-1} \sigma^{i} .
$$


Now

$$
\begin{aligned}
& M\left(y_{m+s+1}, y_{m}, t\right) \\
& \geq M\left(y_{m+s+1}, y_{m}, t \sum_{i=m-1}^{m+s-1} \sigma^{i}\right) \\
& \geq M\left(y_{m+s+1}, y_{m}, t \sigma^{m-1}+t \sigma^{m-1+1}+t \sigma^{m-1+2}+\cdots+t \sigma^{m-1+s}\right) \\
& \geq M\left(y_{m+s+1}, y_{m}, t \sigma^{m-1+1}+t \sigma^{m-1+2}+\cdots+t \sigma^{m-1+s}+t \sigma^{m-1}\right) \\
& \geq \Delta\left(M\left(y_{m+s+1}, y_{m+1}, t \sigma^{m-1+1}+t \sigma^{m-1+2}+\cdots+t \sigma^{m-1+s}\right)\right. \text {, } \\
& \left.M\left(y_{m+1}, y_{m}, t \sigma^{m-1}\right)\right) \\
& \geq \Delta\left(\Delta \left(M\left(y_{m+s+1}, y_{m+2}, t \sigma^{m-1+2}+\cdots+t \sigma^{m-1+s}\right),\right.\right. \\
& \left.\left.M\left(y_{m+2}, y_{m+1}, t \sigma^{m}\right), M\left(y_{m+1}, y_{m}, t \sigma^{m-1}\right)\right)\right) \\
& \geq \Delta\left(\Delta \left(\Delta \left(M\left(y_{m+s+1}, y_{m+3}, t \sigma^{m-1+3}+\cdots+t \sigma^{m-1+s}\right),\right.\right.\right. \\
& \left.\left.\left.M\left(y_{m+3}, y_{m+2}, t \sigma^{m+1}\right), M\left(y_{m+2}, y_{m+1}, t \sigma^{m}\right), M\left(y_{m+1}, y_{m}, t \sigma^{m-1}\right)\right)\right)\right) \\
& \geq \cdots \\
& \geq \overbrace{\Delta(\Delta(\cdots)(\Delta}^{\text {s-times }}\left(M\left(y_{m+s+1}, y_{m+s}, t \sigma^{m-1+s}\right), M\left(y_{m+s}, y_{m+s-1}, t \sigma^{m+s-2}\right),\right. \\
& \left.\left.\left.\left.\cdots, M\left(y_{m+1}, y_{m}, t \sigma^{m-1}\right)\right)\right) \cdots\right)\right) \\
& \geq \overbrace{\Delta(\Delta(\cdots)}^{\text {s-times }}\left(M\left(y_{1}, y_{2}, t \sigma^{m-1+s} / q^{m-1+s}\right), M\left(y_{1}, y_{2}, t \sigma^{m-2+s} / q^{m-2+s}\right)\right. \text {, } \\
& \left.\left.\left.\left.\cdots, M\left(y_{1}, y_{2}, t \sigma^{m-1} / q^{m-1}\right)\right)\right) \cdots\right)\right)
\end{aligned}
$$

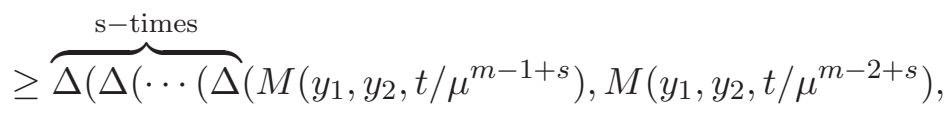

$$
\begin{aligned}
& \left.\left.\left.\left.\cdots, M\left(y_{1}, y_{2}, t / \mu^{m-1}\right)\right)\right) \cdots\right)\right) \\
& \geq \Delta_{i=m-1}^{m+s-1} M\left(y_{1}, y_{2}, t / \mu^{i}\right) \\
& \geq \Delta_{i=m-1}^{\infty} M\left(y_{1}, y_{2}, t / \mu^{i}\right) \text {. }
\end{aligned}
$$

It is obvious that

$$
\lim _{n \rightarrow \infty} \Delta_{i=n}^{\infty} M\left(y_{1}, y_{2}, 1 / \mu^{i}\right)=1 \quad \text { implies } \quad \lim _{n \rightarrow \infty} \Delta_{i=n}^{\infty} M\left(y_{1}, y_{2}, t / \mu^{i}\right)=1
$$

for every $t>0$. Now for every $t>0$ and $\lambda \in(0,1)$, there exists $m_{1}(t, \lambda)$ such that $M\left(y_{m+s+1}, y_{m}, t\right)>1-\lambda$ for every $m \geq m_{1}(t, \lambda)$ and $s \in \mathbb{N}$. Hence $\left\{y_{n}\right\}$ is a Cauchy sequence in $X$. Since $X$ is complete, there exists a point $z$ in $X$ such that $\lim _{n \rightarrow \infty} y_{n}=z$ and this gives

$$
\lim _{n \rightarrow \infty} S x_{2 n}=\lim _{n \rightarrow \infty} T x_{2 n-1}=\lim _{n \rightarrow \infty} A x_{2 n-2}=\lim _{n \rightarrow \infty} B x_{2 n-1}=z
$$


for all $n \in \mathbb{N}$. Without loss of generality, we assume that $S(X)$ is a closed subset of $X$. Then $z=S u$ for some $u \in X$. Subsequently, we have

$$
\lim _{n \rightarrow \infty} S x_{n}=\lim _{n \rightarrow \infty} T x_{n}=\lim _{n \rightarrow \infty} A x_{n}=\lim _{n \rightarrow \infty} B x_{n}=z=S u .
$$

Next, we claim that $A u=S u$.

For this purpose, if we put $x=u$ and $y=x_{n}$ in $(C 3)$, then this gives

$$
\begin{aligned}
1 \leq & F\left(M\left(A u, B x_{n}, q t\right), M\left(S u, T x_{n}, t\right), M(A u, S u, t),\right. \\
& \left.M\left(B x_{n}, T x_{n}, q t\right), M\left(A u, T x_{n}, t\right), M\left(B x_{n}, S u,(q+1) t\right)\right) \\
\leq & F\left(M\left(A u, B x_{n}, q t\right), M\left(S u, T x_{n}, t\right), M(A u, S u, t),\right. \\
& M\left(B x_{n}, T x_{n}, q t\right), M\left(A u, T x_{n}, t\right), \\
& \left.\Delta\left(M\left(B x_{n}, A u, q t\right), M(A u, S u, t)\right)\right) .
\end{aligned}
$$

Taking limit as $n \rightarrow \infty$, we have

$$
\begin{gathered}
1 \leq F(M(A u, z, q t), M(z, z, t), M(A u, z, t), M(z, z, q t), \\
M(A u, z, t), \Delta(M(A u, z, q t), M(A u, z, t)) .
\end{gathered}
$$

Hence we have $M(A u, z, q t), 1) \geq M(A u, z, t)$ for all $t>0$, by Lemma 1.16, we have $A u=S u=z$. Since $A(X) \subset T(X)$, there exists a point $v \in X$ such that $A u=z=T v$.

Next, we claim that $T v=B v$.

Putting $x=u$ and $y=v$ in $(C 3)$, we have

$$
\begin{aligned}
1 \leq & F(M(A u, B v, q t), M(S u, T v, t), M(A u, S u, t), \\
& M(B v, T v, q t), M(A u, T v, t), M(B v, S u,(q+1) t)) \\
= & F(M(A u, B v, q t), M(z, z, t), M(z, z, t), \\
& M(B v, T v, q t), M(z, z, t), M(B v, S u,(q+1) t)) \\
\leq & F(M(A u, B v, q t), 1,1, M(B v, T v, q t), 1, \\
& \Delta(M(B v, A u, q t), M(A u, S u, t))) .
\end{aligned}
$$

Therefore we obtain that

$$
F(M(A u, B v, q t), 1,1, M(B v, A u, q t), 1, M(B v, A u, q t)) \geq 1,
$$

by $F \in \mathcal{F}$, we have $M(B v, T v, q t)) \geq 1$ for all $t>0$ implies that $T v=B v$. Thus $A u=S u=T v=B v=z$. Since the pairs $(A, S)$ and $(B, T)$ are weakly compatible and $u$ and $v$ are their coincidence points, respectively, we obtain $A z=A(S u)=S(A u)=S z$ and $B z=B(T v)=T(B v)=T z$. 
Now, we prove that $z$ is a common fixed point of $A, B, S$ and $T$.

For this purpose, putting $x=z$ and $y=v$ in $(C 3)$, we get

$$
\begin{gathered}
1 \leq F(M(A z, B v, q t), M(S z, T v, t), M(A z, S z, t) \\
M(B v, T v, q t), M(A z, T v, t), M(B v, S z,(q+1) t)) \\
\leq F(M(A z, B v, q t), M(S z, T v, t), M(A z, S z, t), M(B v, T v, q t), \\
M(A z, T v, t), \Delta(M(B v, A z, q t), M(A z, S z, t))) .
\end{gathered}
$$

Again we note that

$$
\begin{gathered}
F(M(A z, B v, q t), M(S z, T v, t), 1,1, \\
M(A z, T v, t), M(B v, A z, q t)) \geq 1,
\end{gathered}
$$

by $F \in \mathcal{F}$, we have $M(A z, B v, q t)) \geq M(S z, T v, t)$ for all $t>0$, by Lemma 1.16, we get $A z=B v$. Hence $A z=B v=z$. Hence $z=A z=S z$ and $z$ is a common fixed point of $A$ and $S$. One can prove that $B v=z$ is also a common fixed point of $B$ and $T$.

Finally, in order to prove the uniqueness, suppose that $w(z \neq w)$ be another fixed point of $A, B, S$ and $T$. Then, for all $t>0$, we have

$$
\begin{array}{rl}
1 \leq F & (M(A z, B w, q t), M(S z, T w, t), M(A z, S z, t), \\
& M(B w, T w, q t), M(A z, T w, t), M(B w, S z,(q+1) t)) \\
\leq F & M(A z, B w, q t), M(S z, T w, t), M(A z, S z, t), \\
& M(B w, T w, q t), M(A z, T w, t), \\
& \Delta(M(B w, A z, q t), M(A z, S z, t))) .
\end{array}
$$

Therefore we have

$$
\begin{gathered}
F(M(A z, B w, q t), M(S z, T w, t), 1,1, \\
M(A z, T w, t), M(B w, A z, q t)) \geq 1 .
\end{gathered}
$$

Hence we have $M(A z, B w, q t)) \geq M(S z, T w, t)$ for all $t>0$, by Lemma 1.16, we get $A z=B w$. Hence $z=w$. This completes the proof.

Next, we prove a fixed point theorem for weakly compatible mappings with E.A. property.

Theorem 2.2. Let $(X, M, \Delta)$ be a complete fuzzy metric space with continuous t-norm of Hadžić-type. Let $A, B, S$ and $T$ be self-mappings on $X$ satisfying $(C 1)-(C 4)$ and the following condotion:

$(C 5)$ the pairs $(A, S)$ or $(B, T)$ satisfy E.A. property.

Then $A, B, S$ and $T$ have a unique common fixed point in $X$. 
Proof. Without loss of generality, we assume that the pair $(B, T)$ satisfies the E.A. property. Then there exists a sequence $\left\{x_{n}\right\}$ in $X$ such that $\lim _{n \rightarrow \infty} B x_{n}=\lim _{n \rightarrow \infty} T x_{n}=z$ for some $z \in X$. Since $B(X) \subset S(X)$, there exists a sequence $\left\{y_{n}\right\}$ in $X$ such that $B x_{n}=S y_{n}$. Hence $\lim _{n \rightarrow \infty} S y_{n}=z$. Also $A(X) \subset T(X)$, there exists a sequence $\left\{y_{n}^{\prime}\right\}$ in $X$ such that $A y_{n}^{\prime}=T x_{n}$. Hence $\lim _{n \rightarrow \infty} A y_{n}^{\prime}=z$. Suppose that $S(X)$ is a closed subset of $X$. Then $z=S u$ for some $u \in X$. Subsequently, we have

$$
\lim _{n \rightarrow \infty} B x_{n}=\lim _{n \rightarrow \infty} T x_{n}=\lim _{n \rightarrow \infty} A y_{n}^{\prime}=\lim _{n \rightarrow \infty} S y_{n}=z=S u
$$

for some $u \in X$.

Next, we claim that $A u=S u$.

For this purpose, if we put $x=u$ and $y=x_{n}$ in (C3), then this gives

$$
\begin{aligned}
1 \leq & F\left(M\left(A u, B x_{n}, q t\right), M\left(S u, T x_{n}, t\right), M(A u, S u, t),\right. \\
& \left.M\left(B x_{n}, T x_{n}, q t\right), M\left(A u, T x_{n}, t\right), M\left(B x_{n}, S u,(q+1) t\right)\right) \\
\leq & F\left(M\left(A u, B x_{n}, q t\right), M\left(S u, T x_{n}, t\right), M(A u, S u, t),\right. \\
& M\left(B x_{n}, T x_{n}, q t\right), M\left(A u, T x_{n}, t\right), \\
& \left.\Delta\left(M\left(B x_{n}, A u, q t\right), M(A u, S u, t)\right)\right)
\end{aligned}
$$

since the function $F$ is non-increasing in the 6-th coordinate variable. Taking limit as $n \rightarrow \infty$, we have

$$
\begin{aligned}
& F(M(A u, z, q t), M(z, z, t), M(A u, z, t), M(z, z, q t), M(A u, z, t), \\
& \quad \Delta(M(A u, z, q t), M(A u, z, t))) \geq 1 .
\end{aligned}
$$

Thus we obtain

$$
\begin{gathered}
F(M(A u, z, q t), 1, M(A u, z, t), 1, M(A u, z, t), \\
\Delta(M(A u, z, q t), M(A u, z, t))) \geq 1,
\end{gathered}
$$

by $F \in \mathcal{F}$, we have $M(A u, z, q t), 1) \geq M(A u, z, t)$ for all $t>0$, by Lemma 1.16 , we get $A u=S u=z$. Since $A(X) \subset T(X)$, there exists a point $v \in X$ such that $A u=z=T v$.

Next, we claim that $T v=B v$.

Putting $x=u$ and $y=v$ in $(C 3)$, we have

$$
\begin{aligned}
1 \leq & F(M(A u, B v, q t), M(S u, T v, t), M(A u, S u, t), \\
& M(B v, T v, q t), M(A u, T v, t), M(B v, S u,(q+1) t)) \\
= & F(M(A u, B v, q t), M(z, z, t), M(z, z, t), \\
& M(B v, T v, q t), M(z, z, t), M(B v, S u,(q+1) t)) \\
\leq & F(M(A u, B v, q t), 1,1, M(B v, T v, q t), 1, \\
& \Delta(M(B v, A u, q t), M(A u, S u, t))) .
\end{aligned}
$$


Therefore we have

$$
F(M(A u, B v, q t), 1,1, M(B v, T v, q t), 1, M(B v, A u, q t)) \geq 1 .
$$

Hence we get $M(B v, T v, q t)) \geq 1$ for all $t>0$ implies that $T v=B v$. Thus $A u=S u=T v=B v=z$. Since the pairs $(A, S)$ and $(B, T)$ are weakly compatible and $u$ and $v$ are their coincidence points, respectively, we obtain $A z=A(S u)=S(A u)=S z$ and $B z=B(T v)=T(B v)=T z$.

Now, we prove that $z$ is a common fixed point of $A, B, S$ and $T$.

For this purpose, if we put $x=z$ and $y=v$ in $(C 3)$, then this gives

$$
\begin{aligned}
1 \leq & F(M(A z, B v, q t), M(S z, T v, t), M(A z, S z, t), \\
& M(B v, T v, q t), M(A z, T v, t), M(B v, S z,(q+1) t)) \\
\leq F & (M(A z, B v, q t), M(S z, T v, t), M(A z, S z, t), \\
& M(B v, T v, q t), M(A z, T v, t), \\
\Delta & (M(B v, A z, q t), M(A z, S z, t))) .
\end{aligned}
$$

Therefore we have

$$
\begin{gathered}
F(M(A z, B v, q t), M(S z, T v, t), 1,1 \\
M(A z, T v, t), M(B v, A z, q t)) \geq 1 .
\end{gathered}
$$

Hence we get $M(A z, B v, q t)) \geq 1$ for all $t>0$ implies that $A z=B v$ and hence $A z=B v=z$. Therefore $z=A z=S z$ and $z$ is a common fixed point of $A$ and $S$. One can easily prove that $B v=z$ is also a common fixed point of $B$ and $T$.

Finally, in order to prove the uniqueness, let $w(z \neq w)$ be another fixed point of $A, B, S$ and $T$. Then, for all $t>0$, we have

$$
\begin{aligned}
1 \leq F & (M(A z, B w, q t), M(S z, T w, t), M(A z, S z, t), \\
& M(B w, T w, q t), M(A z, T w, t), M(B w, S z,(q+1) t)) \\
\leq F & (M(A z, B w, q t), M(S z, T w, t), M(A z, S z, t), \\
& M(B w, T w, q t), M(A z, T w, t), \\
& \Delta(M(B w, A z, q t), M(A z, A z, t))) .
\end{aligned}
$$

Therefore we have

$$
\begin{gathered}
F(M(A z, B w, q t), M(S z, T w, t), 1,1 \\
M(A z, T w, t), M(B w, A z, q t)) \geq 1 .
\end{gathered}
$$

Hence we get $M(A z, B w, q t)) \geq 1$ for all $t>0$ implies that $A z=B w$, i.e., $z=w$. This completes the proof. 
Finally, we prove a fixed poin theorem for weakly compatible mappings with CLRs property.

Theorem 2.3. Let $(X, M, \Delta)$ be a fuzzy metric space with continuous $t$-norm of Hadžić-type. Let $A, B, S$ and $T$ be self-mappings on $X$ satisfying $(C 1),(C 2),(C 3)$ and the following conditions:

(C6) the pairs $(A, S)$ or $(B, T)$ satisfy CLRs property,

$(C 7)$ one of subspaces $A(X), B(X), S(X)$ or $T(X)$ is closed of $X$.

Then $A, B, S$ and $T$ have a unique common fixed point in $X$.

Proof. If the pair $(A, S)$ satisfies the CLRs property, then there exists a sequence $\left\{x_{n}\right\}$ in $X$ such that $\lim _{n \rightarrow \infty} A x_{n}=\lim _{n \rightarrow \infty} S x_{n}=z$, where $z \in S(X)$. Therefore there exists a point $u \in X$ such that $S u=z$. Since $T(X)$ is closed of $X$ and $A(X) \subset T(X)$, so for each $\left\{x_{n}\right\}$ in $X$, there corresponds a sequence $\left\{y_{n}\right\}$ in $X$ such that $A x_{n}=T y_{n}$. Therefore $\lim _{n \rightarrow \infty} T y_{n}=\lim _{n \rightarrow \infty} A x_{n}=z$, where $z \in S(X)$. Thus we have $\lim _{n \rightarrow \infty} A x_{n}=\lim _{n \rightarrow \infty} S x_{n}=\lim _{n \rightarrow \infty} T y_{n}=z$.

Now, we are required to show that $\lim _{n \rightarrow \infty} B y_{n}=z$.

Putting $x=x_{n}$ and $y=y_{n}$ in $(C 3)$, we get

$$
\begin{aligned}
& F\left(M\left(A x_{n}, B y_{n}, q t\right), M\left(S x_{n}, T y_{n}, t\right), M\left(A x_{n}, S x_{n}, t\right),\right. \\
& \left.\quad M\left(B y_{n}, T y_{n}, q t\right), M\left(A x_{n}, T y_{n}, t\right), M\left(B y_{n}, S x_{n},(q+1) t\right)\right) \geq 1 .
\end{aligned}
$$

We assume that $\lim _{n \rightarrow \infty} B y_{n}=l \neq z$ for $t>0$. Then taking limit as $n \rightarrow \infty$, we have

$$
\begin{aligned}
1 \leq & F(M(z, l, q t), M(z, z, t), M(z, z, t), \\
& M(l, z, q t), M(z, z, t), M(l, z,(q+1) t)) \\
= & F(M(z, l, q t), 1,1, M(l, z, q t), 1, M(l, z,(q+1) t)) \\
\leq & F(M(z, l, q t), 1,1, M(l, z, q t), 1, \Delta(M(l, z, q t), M(z, z, t)))
\end{aligned}
$$

since the function $F$ is non-increasing in the 6 -th coordinate variable. Therefore we have

$$
F(M(z, l, q t), 1,1, M(l, z, q t), 1,(M(l, z, q t)) \geq 1,
$$

by $F \in \mathcal{F}$, we get $M(z, l, q t) \geq 1$ implies that $z=l$ and hence $\lim _{n \rightarrow \infty} B y_{n}=z$. Therefore

$$
\lim _{n \rightarrow \infty} A x_{n}=\lim _{n \rightarrow \infty} S x_{n}=\lim _{n \rightarrow \infty} T y_{n}=\lim _{n \rightarrow \infty} B y_{n}=z=S u
$$

for some $u \in X$. Using Theorem 2.1 and implicit relations $\mathcal{F}$, we can easily prove that $z$ is a unique common fixed point of $A, B, S$ and $T$. This completes the proof. 


\section{Acknowledgments}

The fourth author (Professor Sanjay Kumar) would like to acknowledge UGC for providing Major Research Project under Ref.(39-41/2010(SR)).

\section{References}

[1] M. Aamri, D. El Moutawakil, Some new common fixed point theorems under strict contractive conditions, J. Math. Anal. Appl., 270, No. 1 (2002), 181-188, doi: 10.1016/S0022-247X(02)00059-8.

[2] Z. Deng, Fuzzy pseudo-metric space, J. Math. Anal. Appl., 86, No. 1 (1982), 74-95, doi: 10.1016/0022-247X(82)90255-4.

[3] M.A. Erceg, Metric space in fuzzy set theory, J. Math. Anal. Appl., 69, No. 1 (1979), 205-230, doi: 10.1016/0022-247X(79)90189-6.

[4] A. George and P. Veeramani, On some results in fuzzy metric spaces, Fuzzy Sets and Systems, 64, No. 3 (1994), 395-399, doi: 10.1016/01650114(94)90162-7.

[5] M. Grabiec, Fixed points in fuzzy metric space, Fuzzy Sets Syestes, 27, No. 3 (1988), 385-389, doi: 10.1016/0165-0114(88)90064-4.

[6] O. Hadžić, A fixed point theorem in Menger spaces, Publ. Inst. Math. (Beograd) (N.S.), 26(40) (1979), 107-112.

[7] O. Hadžić and E. Pap, Fixed Point Theory in Probabilistic Metric Spaces, Kluwer Academic Publishers, Dordrechi (2001).

[8] M. Imdad and J. Ali, Some common fixed point theorems in fuzzy metric spaces, Math. Commun., 11, No. 2 (2006), 153-163.

[9] M. Imdad and J. Ali, Jungcks common fixed point theorem and E.A. property, Acta Math. Sin. (Engl. Ser.), 24, No. 1 (2008), 87-94, doi: 10.1007/s10114-007-0990-0.

[10] G. Jungck, Commuting mappings and fixed points, Amer. Math. Monthly, 83, No. 4 (1976), 261-163, doi: 10.2307/2318216.

[11] G. Jungck, Common fixed points for noncontinuous nonself mappings on non metric spaces, Far East J. Math. Sci., 4, No. 2 (1996), 199-215. 
[12] O. Kaleva and S. Seikkala, On fuzzy metric spaces, Fuzzy Sets and Systems, 12, No. 1 (1984), 215-229, doi: 10.1016/0165-0114(84)90069-1.

[13] I. Kramosil and J. Michalek, Fuzzy metrics and statistical metric spaces, Kybernetika, 11, No. 5 (1975), 336-344.

[14] S. Kumar, Fixed point theorems for weakly compatible maps under E.A. property in fuzzy metric spaces, J. Appl. Math. Inform., 29, No. 1-2 (2011), 395-405.

[15] S.N. Mishra, N. Sharma and S.L. Singh, Common fixed points of maps on fuzzy metric spaces, Internat. J. Math. Math. Sci., 17, No. 2 (1994), 253-258, doi: 10.1155/S0161171294000372.

[16] R.P. Pant, Common fixed point for noncommuting mapping, J. Math. Anal. Appl., 188, No. 2 (1994), 436-440, doi: 10.1006/jmaa.1994.1437.

[17] W. Sintunavarat and P. Kumam, Common fixed point theorems for a pair of weakly compatible mappings in fuzzy metric spaces, J. Appl. Math., 2011 (2011), Article ID 637958, 14 pages, doi: 10.1155/2011/637958.

[18] P.V. Subrahmanyam, Common fixed point theorems in fuzzy metric spaces, Inform. Sci., 83, No. 3-4 (1995), 109-112, doi: 10.1016/00200255(94)00043-B.

[19] R. Vasuki, Common fixed points for weakly commuting maps in fuzzy metric spaces, Indian J. Pure Appl. Math., 30, No. 4 (1999), 419-423.

[20] L.A. Zadeh, Fuzzy sets, Inform. Control, 18 (1965), 338-353. 
